\title{
Abnormal behaviors during sleep from the viewpoint of sleep epileptology: current and future perspectives on diagnosis
}

\author{
Shigeru Chiba
}

\begin{abstract}
Abnormal behaviors during sleep (ABDS) exhibit a myriad of symptoms. Their underlying diseases are also diverse, which include NREM/REM-related parasomnias, epilepsy and mental disorders. Since ABDS may severely affect a patient's quality of life, giving an early and accurate diagnosis of the underlying disease (by analyzing videopolysomnographic data during the manifestation of ABDS) is of great importance. However, accurate diagnosis of ABDS is rather difficult. Recently it has been suggested that the pathology of (NREM/REM-related) parasomnias and epilepsy are closely related. In order to unravel the pathophysiological substrate of ABDS, it is essential to develop a novel approach based on sleep epileptology, a field which targets the interface between sleep medicine and epileptology.
\end{abstract}

Keywords: Sleep, Parasomnias, Epilepsy

\section{Background}

Abnormal behaviors during sleep (ABDS) can range across a wide spectrum, from simple and minor motor activities (e.g., paroxysmal arousals, limb myoclonus) to complex and intense behaviors (e.g., wandering, talking, screaming, hyperactivity, violence).

Diagnosis of ABDS is difficult for the following reasons (Breen et al. 2018; Ingravallo et al. 2014). On the patient side, i) since ABDS are rarely observed (or video recorded), patients have little or no subjective information about the symptoms (it is also often the case that nobody else notices them); ii) patients do not have a medical examination until they realize disadvantages in social life, or experience trauma caused by ABDS. On the doctor side, i) although video-polysomnography (VPSG) using full-montage electroencephalography (EEG) is a highly regarded test, it requires a lot of time and money. Only a few patients take V-PSG; ii) the number of medical specialists who can accurately diagnose ABDS is still low.

Correspondence: chibas@asahikawa-med.ac.jp

Department of Psychiatry and Neurology, School of Medicine, Asahikawa

Medical University, Midorigaoka higashi 2-1-1-1, Asahikawa, Hokkaido 078-8510, Japan
Patients suffer from ABDS not only at night. ABDS cause inadequate sleep quantity and quality at night, which in turn causes excessive daytime sleepiness. ABDS tend to result in functional disorders in various situations that include a patient's family life, social life, career plan, and school life. Due to ABDS, patients and their bed partners can even get hurt, which sometimes requires forensic psychiatric evidence/decisions (Ingravallo et al. 2014). In short, ABDS may severely affect the quality of life (QOL) of patients and their families (Breen et al. 2018; Ingravallo et al. 2014).

In this paper, I propose a clinical-practice based classification of the underlying diseases of ABDS and present perspectives from some of the best research on pathophysiological relations between parasomnias and epilepsy, both representing typical ABDS. I also argue that it is imperative to develop a novel approach based on sleep epileptology (Chiba 2019), a field which targets the interface between sleep medicine and epileptology to uncover the pathophysiology behind ABDS.

\section{Underlying diseases of ABDS}

The underlying diseases of ABDS are classified into two categories (Table 1): i) Sleep disorders and ii) Psychiatric disorders. As for the first category, I adapted the recent

(c) The Author(s). 2020 Open Access This article is distributed under the terms of the Creative Commons Attribution 4.0 International License (http://creativecommons.org/licenses/by/4.0/), which permits unrestricted use, distribution, and 
Table 1 Major underlying diseases of abnormal behaviors during sleep (ABDS)

\begin{tabular}{|c|c|}
\hline Disease categories & Representative ABDS \\
\hline \multicolumn{2}{|l|}{ Sleep disorders ${ }^{\mathrm{a}}$} \\
\hline Insomnia & movements associated with sleep arousals \\
\hline Sleep-related breathing disorders & occasional flailing movements associated with apneic arousals \\
\hline Central disorders of hypersomnolence & narcolepsy with "negative" and "active" movement abnormalities \\
\hline Parasomnias & NREM/REM parasomnias, status dissociatus \\
\hline Sleep-related movement disorders & $\begin{array}{l}\text { restless legs syndrome, periodic limb movement disorder, sleep-related leg cramp, sleep-related bruxism, } \\
\text { fasciomandibular myoclonus, sleep-related rhythmic movement disorder, benign sleep myoclonus of } \\
\text { infancy, propriospinal myoclonus at sleep onset }\end{array}$ \\
\hline $\begin{array}{l}\text { Sleep-related medical and neurological } \\
\text { disorders }\end{array}$ & sleep-related epilepsy \\
\hline Other sleep disorders & anti-IgLON5 disease, ADCY5-associated disease, benign nocturnal alternating hemiplegia of childhood \\
\hline Isolated symptoms or normal variants & $\begin{array}{l}\text { excessive fragmentary myoclonus, hypnagogic foot tremor, alternating leg muscle activation, high } \\
\text { frequency leg movements, hypnic jerk, neck myoclonus during sleep }\end{array}$ \\
\hline \multicolumn{2}{|l|}{ Psychiatric disorders } \\
\hline Delirium & consciousness disturbance, hyperactivity, nocturnal insomnia, sleep-wake cycle disturbance, nightmare \\
\hline Panic disorder & panic attacks during NREM sleep \\
\hline Posttraumatic stress disorder & insomnia, nightmare \\
\hline Psychogenic nonepileptic seizures & epileptic seizure-like symptoms during waking \\
\hline Dissociative (conversion) disorders & amnesia, fugue, stupor, motor disorders, convulsion \\
\hline
\end{tabular}

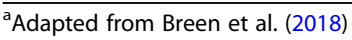

classification of Breen et al. (Breen et al. 2018) who incorporated the latest insights into the International Classification of Sleep Disorders (ICSD), third edition (ICSD-3, 2014) (American Academy of Sleep Medicine 2014). In the other category, from my clinical experience, of particular importance are delirium, panic disorder, posttraumatic stress disorder (PTSD), psychogenic nonepileptic seizures (PNES) and dissociative (conversion) disorder, which are frequently observed in medical practice. Hence we always bear them uppermost in mind when we make a differential diagnosis of ABDS.

Delirium has transient consciousness disturbance as its cardinal symptom, frequently observed in inpatients at general hospitals (10-82\%) (Inouye et al. 2014). Hyperactive delirium requires prompt clinical treatment. Possible hyperactive delirium could sometimes turn out to be ictal/postictal delirium caused by epileptic seizures. It is frequently observed that elderly patients manifest nocturnal insomnias, sleep-wake cycle disturbance, and nightmares before delirium (Hatta et al. 2017). It is reported that early treatment of these sleep disorders could prevent development of delirium (Hatta et al. 2017).

Most patients with panic disorders have experienced panic attacks not only during the daytime but also at night (Staner 2003). Panic attacks are sometimes wrongly diagnosed as sleep terrors, nightmare disorders, or epilepsy. V-PSG observation at the manifestation of panic attacks indicates that panic attacks are likely to occur during the transitional period from stage 2 to stage 3 of non-REM
(NREM) sleep but not while awake or during REM sleep (Staner 2003). On the other hand, parasomnias and nightmare disorders occur during stage 4 of NREM sleep and REM sleep, respectively (Staner 2003).

People with PTSD show a high incidence of sleep disorders. A study in the United States investigated 277 adult patients with PTSD and reported that about $93 \%$ of the patients had also developed sleep disorders: $56.7 \%$ had both insomnias and nightmares, $24.9 \%$ had only insomnias and $11.3 \%$ had only nightmares (Milanak et al. 2019). Patients without sleep disorders accounted for just 6.9\% (Milanak et al. 2019). Note that PTSD may also cause dream enactment and parasomnias (Breen et al. 2018).

PNES are characterized by sudden and time-limited disturbances of motor, sensory, autonomic, cognitive, and/or emotional functions that are often misdiagnosed as epileptic seizures. Although PNES mostly occur during the day, they can occur at any time during the night. PNES always occur during awakening but never during sleep. In contrast to epileptic seizures, PNES are not associated with epileptiform discharges seen in EEG, but are instead derived from psychologic underpinnings (Chen et al. 2017; Gates et al. 1985). Intractable epilepsy is complicated by PNES at high rates. Among patients referred to outpatient epilepsy centers, 5 to $25 \%$ are considered to have PNES, while 25 to $40 \%$ of patients evaluated in inpatient epilepsy monitoring units for intractable seizures are diagnosed with PNES (Chiba 2019; Szaflarski et al. 2000). Since patients with intractable epilepsy develop high-frequent 
PNES, it is important to make an accurate differential diagnosis differentiating true seizures from PNES.

Dissociative (conversion) disorders also manifest various ABDS that may resemble epileptic seizures and PNES. Dissociative (conversion) disorders are considered to underlie most PNES (Lawton et al. 2008).

Table 1 gives a list of potential underlying diseases of ABDS. In actual medical practice, patients suffering from ABDS may have multiple underlying diseases (e.g., parasomnias and PTSD, sleep-related epilepsy and PNES).

\section{Parasomnias and epilepsy}

\section{Significance of V-PSG and stereo-EEG}

The introduction of V-PSG into clinical practice in the 1980's has enabled highly accurate differential analysis for ABDS. Furthermore, it has played an important role in detecting a large body of useful clinical information for treating patients, which includes abnormal sleep architecture, sleep disorders (such as insomnias and sleep apneas), epileptic seizures and interictal/ictal epileptiform discharges which would often be overlooked in the daytime. For instance, the incidence rate of subjective sleep disorders was high among epileptic patients, at approximately $40 \%$ (van Golde et al. 2011), and the disorders have been confirmed by several V-PSG diagnoses such as degradation in the quantity and quality of nocturnal sleep. Studies of generalized epilepsy, for instance, have reported reduction in the percentage of NREM sleep (Krishnan et al. 2014), reduction of REM sleep (Mekky et al. 2017; Roshan et al. 2017) and increased time awake after sleep onset (Krishnan et al. 2014; Mekky et al. 2017) in comparison to control populations. In focal epilepsy, there are reports of decreased REM sleep (Parrino et al. 2012a), decreased (Miller et al. 2016) or increased N3 (Parrino et al. 2012a) and increased time awake after sleep onset (Parrino et al. 2012a). However, only a few studies objectively assessed sleep in adults with epilepsy while controlling for key factors that influence sleep (SudbrackOliveira et al. 2019). Further studies are required to access the extent of sleep architectural abnormalities in adults with epilepsy (Sudbrack-Oliveira et al. 2019).

V-PSG is a highly regarded test in making a differential diagnosis between parasomnias and epilepsy. V-PSG has to simultaneously document video-recorded behaviors, as well as record electrooculograms, electromyograms, and various other biological phenomena. In order to give a differential diagnosis of ABDS, the following information is crucial: under which brain conditions (awakening, NREM sleep, or REM sleep) and under what other conditions (facial expression, body movement, circulatory and respiratory dynamics, etc.) did ABDS occur? We usually continuously record V-PSG using full-montage EEG for 1 to 3 days (9-72 h). Other electrodes such as sphenoidal electrodes may be added to the full-montage electrodes depending on diagnostic purposes.

Depth electrodes or subdural electrodes may be implanted into the brains of patients with intractable epilepsy, who are potential candidates to receive neurosurgery, as an examination before operation. EEG in VPSG performed for such patients are called stereo-EEG (S-EEG) [that is based on the stereotactic placement of a number of intracerebral multilead electrodes to obtain long-term EEG recording in a 3-D arrangement] (Gibbs et al. 2016). S-EEG can be an invasive test in brain tissues and hence it should be allowed only as an examination before epileptic surgery. Although S-EEG has been used in very restricted settings, it offers precious opportunities to observe how a patient's deep brain structure is involved in epileptic seizures and various ABDS (Gibbs et al. 2016).

\section{Sleep-related epilepsy}

Janz (1974) examined 2825 epilepsy patients with generalized tonic-chronic seizures and investigated the association between the seizures and the sleep/wake cycle through clinical observation. Symptoms were classified into three groups: sleep epilepsy with seizures occurring during sleep (44\%), awakening epilepsy with seizures occurring shortly after awakening (33\%), and diffuse epilepsy with seizures occurring with no correlation to the sleep/waking cycle (23\%).

The term sleep-related epilepsy covers both sleep epilepsy (e.g., nocturnal frontal lobe epilepsy) and awakening epilepsy (e.g., juvenile myoclonic epilepsy). Although it was not in ICSD-1 (1990) (American Sleep Disorders Association (Thorpy MJ, Chairman) 1990), it was mentioned in both ICSD-2 (2005) (American Academy of Sleep Medicine 2005) and ICSD-3 (2014) (American Academy of Sleep Medicine 2014). According to Janz (1974), sleep-related epilepsy, which includes both sleep epilepsy (44\%) and awakening epilepsy (33\%), accounts for a total of $77 \%$ of epilepsy incidents. It can be said that epilepsy is a brain disease closely related to sleep.

As we have already seen, Janz (1974) is a pioneer in the clinical study of the association between sleep and epilepsy, providing a conceptual basis for sleep-related epilepsy.

Table 2 summarizes sleep-related epilepsy. Seizures caused by sleep-related hypermotor epilepsy (nocturnal frontal lobe epilepsy), a representative of focal epilepsy, occur mostly during NREM sleep and rarely during REM sleep. This will be discussed in more detail later. The expected reason is that thalamocortical hypersynchrony during NREM sleep promotes epileptic seizure generation (Herman et al. 2001). 
Table 2 Representative sleep-related epilepsy

Sleep epilepsy
Sleep-related hypermotor epilepsy (nocturnal frontal lobe epilepsy)
Temporal lobe epilepsy
Benign epilepsy of children with centrotemporal foci
Benign epilepsy with occipital paroxysms
Lennox-Gastaut syndrome (tonic seizures)
Epilepsy with continuous spikes and waves during slow-wave sleep
Landau-Kleffner syndrome (acquired epileptic aphasia)
Awakening epilepsy
Juvenile myoclonic epilepsy
Epilepsy with generalized tonic-clonic seizures on awakening

From nocturnal paroxysmal dystonia to nocturnal frontal lobe epilepsy

Lugaresi and Cirignotta (1981) described five patients with frequent episodes occurring in clusters during sleep, characterized by bizarre movements and/or dystonic-tonic posturing of the limbs. These patients showed no interictal and ictal epileptiform discharges in scalp EEG but had a good response to carbamazepine. Based on these findings, the authors came to consider the condition an unusual motor disorder of sleep with an unclear pathophysiology, and labeled the disorder "hypnogenic paroxysmal dystonia", modified later to nocturnal paroxysmal dystonia (NPD) (Lugaresi et al. 1986).

Several years later, Wada (Wada and Purves 1984; Wada 1988) reported that pre-surgical S-EEG evaluation in intractable epileptic patients revealed the epileptiform discharges of frontal origin during the NPD seizures. Similar findings were reported by other investigators (Waterman et al. 1987; Williamson et al. 1985). Furthermore, Tinuper et al. (1990) demonstrated clear-cut epileptiform discharges in the ictal and interictal EEG recordings of three patients previously diagnosed as NPD. These findings suggest that NPD is of epileptic origin and the term was changed to the term nocturnal frontal lobe epilepsy (NFLE).

The V-PSG studies on NPD have greatly contributed to the refinement of the criteria in the ICSD. In particular, it should be noted that NPD, which was originally in the category of parasomnias in ICSD-1 (1990) (American Sleep Disorders Association (Thorpy MJ, Chairman) 1990), was reclassified into the category of NFLE in both ICSD-2 (2005) (American Academy of Sleep Medicine 2005) and ICSD-3 (2014) (American Academy of Sleep Medicine 2014), which implies that without V-PSG, it is extremely difficult to make a differential diagnosis of epilepsy from parasomnias.

The clinical boundaries of NFLE have been mostly refined by the Bologna school (Italy) (Provini et al. 1999,
2000; Tinuper et al. 1990, 2002, 2005; Tinuper and Lugaresi 2002). The clinical symptoms of NFLE, which are similar to those of NPD, are as follows: (1) paroxysmal arousals, (2) hypermotor seizures, (3) asymmetric bilateral tonic seizures, and (4) prolonged epileptic nocturnal wanderings (Montagna et al. 1990; Montagna 1992; Plazzi et al. 1995; Tinuper et al. 2005). The clinical spectrum comprises distinct paroxysmal sleep-related seizures of variable duration (3-120 s, or more) and complexity (Tinuper et al. 2002) ranging from paroxysmal arousals or very brief motor attacks to hypermotor seizures sometimes followed by prolonged complex ambulatory behavior.

Provini et al. (1999) examined the clinical and V-PSG findings of 100 consecutive patients with NFLE. NFLE seizures predominate in males (70\%). Age at onset of the nocturnal seizures varies, but centers during infancy and adolescence. A familial recurrence of the epileptic attacks is found in $25 \%$ of the cases, while $39 \%$ of the patients present a family history of nocturnal paroxysmal episodes that fit the diagnostic criteria for parasomnias. Incidence of neuroradiological findings are low. In many patients, ictal (44\%) and interictal (51\%) EEG findings are uninformative. Marked autonomic activation is a common finding during the seizures. NFLE does not show a tendency to spontaneous remission. Carbamazepine completely abolishes the seizures in approximately $20 \%$ of the cases and gives remarkable relief (reduction of the seizures by at least $50 \%$ ) in another $48 \%$.

Note also that a family was reported to have a high incidence of both (NREM or REM-related) parasomnias and nocturnal frontal lobe epilepsy (Tinuper et al. 2010). Therefore, it is suggested that NFLE and parasomnias share some common pathophysiologic substrate, which requires further investigation.

\section{Sleep-related hyperactive epilepsy: a new concept of NFLE}

At a Consensus Conference held in Bologna, Italy in 2014, NFLE was renamed sleep-related hypermotor epilepsy (SHE), based on three critical issues justifying the renaming (Tinuper et al. 2016; Tinuper and Bisulli 2017). First, the term nocturnal was considered misleading because it implies a chronobiological pattern of seizure occurrence, whereas evidence indicates that occurrence in sleep is the most important characteristic rather than the time of day. Second, the term frontal lobe is not always appropriate because the characteristic seizures may also arise from extrafrontal areas. In SHE, approximately $70 \%$ of cases have a frontal lobe origin, whereas the remaining $30 \%$ of the cases have an extrafrontal origin, coming from the insula, the temporal lobe, as well as the parietal lobe. Third, the term NFLE did not specify the typical clinical semiology involved, 
which consists primarily of hypermotor seizures (Tinuper et al. 2016; Tinuper and Bisulli 2017).

SHE diagnosis is primarily based on clinical history. The absence of clear interictal and ictal epileptiform discharges correlates does not necessarily indicate a negative SHE diagnosis. Three different levels of diagnostic certainty have been identified: (1) Witnessed (possible), based on observation by a witness of the core clinical features, but without other sources of evidence. (2) Video-documented (clinical), which involves a high quality video recording of at least one (but preferably two) stereotyped episodes. (3) Video-EEG-documented (confirmed), which requires the V-PSG recording of at least one but preferably two stereotyped events with documented ictal discharge or interictal epileptiform abnormalities (Tinuper et al. 2016; Tinuper and Bisulli 2017).

\section{Sleep and its instability in epilepsy}

Fine EEG changes called Cyclic Alternating Pattern (CAP) in intractable epilepsy, which indicate unstable NREM sleep, have been observed at a high rate among patients with intractable epilepsy (Parrino et al. 2012b). It has also been suggested that CAP (the phase A of the CAP) may trigger epileptic seizures (CAP-related seizures) (Halász et al. 2013; Parrino et al. 2012b). These sleep disorders are caused by epileptic seizures, whereas epileptic seizures can also be caused by the disorders. This mutually advancing relation holds between epileptic seizures and sleep disorders.

Paroxysmal arousals (PAs) and minor motor events (MMEs) are mild motor events seen in NFLE. PAs are characterized by sudden and brief arousals (5-10 s) often accompanied by stereotyped movements, dystonic posture, vocalization, frightened facial expression, and/or fear. MMEs are even shorter (2-4s) movements, often stereotyped, involving the axial musculature or the head and limb (Gibbs et al. 2016). Both PAs and MMEs may go unnoticed by the bed partner or family members (Gibbs et al. 2016). Interictal/ictal epileptiform discharges of PAs and MMEs are often not detectable in scalp EEG recordings (Gibbs et al. 2016).

V-PSG with S-EEG studies revealed that PAs are epileptic seizures because all PAs are associated with epileptiform discharges. However, the same cannot be said of MMEs (Gibbs et al. 2016).

According to a V-PSG with S-EEG study on the relationship between MMEs, epileptiform discharges, and arousal fluctuations during sleep (Terzaghi et al. 2008), the MMEs as well as epileptiform discharges shared a close relationship with arousal fluctuations as depicted by analysis of the CAP, preferably occurring in phase A of the CAP. Therefore, epileptiform discharges-related MMEs seem not to be epileptic seizures but to be nonepileptic motor events which are related to sleep instability (CAP). MMEs may be facilitated, in a nonspecific way, by the presence of epileptiform discharges (Terzaghi et al. 2007).

In summary, PAs seem to be epileptic seizures of NFLE (SHE), while MMEs are not epileptic. Several studies suggest that sleep-related epileptiform discharges internally increase sleep instability (CAP rate) that in turn enhance the occurrence of MMEs or other sleeprelated motor events (e.g., parasomnias, periodic limb movement disorder). Conversely, the increased sleep instability (increased CAP rate) would also facilitate the generation of the sleep-related epileptiform discharges.

\section{Delirium, REM sleep behavior disorder, and epilepsy}

V-PSG may be difficult to carry out in delirious patients because they often exhibit psychomotor excitement. Japanese investigators reported a few V-PSG studies of delirium. This delirium took place during the alcoholic withdrawal period in alcoholics, and during the delirious state immediately after administration of an anticholinergic agent (biperiden) in normal volunteers (Hishikawa 1991; Kojima et al. 2000; Tachibana et al. 1975). These studies revealed peculiar polygraphic readings called stage 1-REM with tonic EMG (stage 1-REM) could be found during delirium. Stage 1-REM is characterized by the features of both stage 1 and stage REM, with concomitant occurrences of low voltage, fast-and-slow mixed frequency EEG, markedly elevated tonic EMG, and markedly increased rapid eye movements (Hishikawa 1991; Tachibana et al. 1975).

It is reported that REM sleep without atonia that is similar to stage 1-REM is observed in REM sleep behavior disorder (RBD) (Ferri et al. 2014; Schenck et al. 1986; Sunwoo et al. 2019) and acute RBD (Provini and Tachibana 2018) that has been associated with various medications or substances, in particular antidepressants, and the abrupt withdrawal from barbiturates, tricyclic antidepressants, monoamine oxidase inhibitors, and alcohol. Therefore, the same pathophysiology of stage 1-REM can be observed not only in delirium but also in RBD and acute RBD.

We confirmed experimentally that the behavioral and video-polygraphic changes induced by biperiden administration in rats are consistent with those of delirium in humans (Tamura et al. 2006). These findings suggest that a biperiden-treated rat is a good animal model for anticholinergic delirium (Tamura et al. 2006). These findings also indicate that anticholinergic mechanisms play an important role in the manifestation of hyperactive delirium, involved with changes in arousal level and REM sleep mechanisms (Tamura et al. 2006).

Recently, Hatta et al. (2017) suggested that in a randomized placebo-controlled study, suvorexant, a potent and selective orexin receptor antagonist, is effective for 
the prevention of delirium in elderly patients admitted for acute care. Because the main effect of suvorexant was seen in the sleep-wake cycle of the Japanese version of the Delirium Rating Scale-Revised-98 (Hatta et al. 2017) and the drug has not shown cholinergic affinities, the reservation/restoration of sleep-wake cycle may have priority over cholinergic neurotransmission in delirium prevention.

Epilepsy may coexist with delirium and RBD. In patients with epilepsy, it is well known that delirium is frequently observed during the ictal and postictal period. Manni et al. (2007) reported that RBD episodes were found to coexist with epilepsy in 10 out of 80 (12.5\%) elderly subjects (aged 60 or over). Iranzo et al. (2006) also reported five patients with potassium channel antibody-associated limbic encephalopathy who showed simple or complex partial seizures of epilepsy with epileptiform discharges in the temporal regions during wakefulness as well as RBD episodes confirmed by VPSG. It has been suggested that REM sleep inhibits epileptic phenomena due to a desynchronization of cortical cellular discharges and the physiological muscle atonia. Interestingly, a number of experimental studies showed that in feline REM sleep without cortical EEG desynchrony and REM sleep without atonia induced pontine dissociation techniques facilitate seizures of both generalized and limbic seizure models (Shouse 2002). Although the pathophysiological mechanisms of the coexistence of RBD and epilepsy is still unclear, functional abnormalities of the limbic system and the brainstem is assumed to be responsible for the coexistence (Iranzo et al. 2006).

\section{Conclusion}

ABDS show a myriad of symptoms ranging from simple and minor motor activities to complex and intense behaviors. Their underlying diseases are also diverse, which include psychiatric disorders not listed in in the ICSD-3 such as delirium, panic disorder, PTSD, PNES and dissociative (conversion) disorder. Since ABDS may severely affect the QOL of patients and their families, correctly giving an early diagnosis of the underlying disease(s) is extremely important. In clinical practice, it is imperative to properly build V-PSG findings during the manifestation of ABDS.

Since the pathophysiology behind ABDS is not fully understood, the present paper focused on parasomnias and epilepsy, the underlying diseases of ABDS, and discussed some important achievements in ABDS research.

The progress of the two research fields of sleep medicine and epileptology has been advancing with the recent development of long-term monitoring using $\mathrm{V}$ PSG with full-montage EEG, various brain imaging technologies, and genetic screening systems. Great attention has been paid to the close relationship between the two fields, accumulating fruitful research findings. S-EEG has deepened our understanding of deep brain structure which scalp EEG could not capture.

Previous studies on V-PSG have made great contributions to the refinement of the criteria in the ICSD-1, -2 , and -3 . In particular, it should be noted that nocturnal paroxysmal dystonia, which was originally in the category of parasomnias in ICSD-1 (1990), was reclassified into the category of NFLE in both ICSD-2 (2005) and ICSD-3 (2014), which implies that without V-PSG, it is extremely difficult to make a differential diagnosis of parasomnias from epilepsy.

The underlying diseases behind ABDS turned out to be more than targets of differential diagnoses and have some pathophysiologic similarity. Recently, fine EEG changes called CAP in intractable epilepsy, which indicate unstable NREM sleep, have been observed at a high rate among patients with intractable epilepsy. It has also been suggested that CAP may trigger epileptic seizures (CAP-related seizures). These sleep disorders are caused by epileptic seizures, whereas epileptic seizures can also be caused by the disorders. This mutual relation holds between epileptic seizures and sleep disorders.

To sum up, it has been suggested that sleep disorders and epilepsy are closely associated with each other. Sleep epileptology, which covers the interface between sleep medicine and epileptology, will enable us to deepen our understanding of the semiology of ABDS, refine the diagnostic criteria, and take further steps towards unraveling the pathophysiology behind ABDS. In order to develop treatments for patients suffering with ABDS and improve their QOL, it is absolutely essential for sleep epileptology to establish its own field by accumulating findings and achievements.

\section{Abbreviations}

(ABDS): abnormal behaviors during sleep; (CAP): cyclic alternating pattern; (EEG): electroencephalography; (ICSD): International Classification of Sleep Disorders; (MMEs): minor motor events; (NFLE): nocturnal frontal lobe epilepsy; (NPD): nocturnal paroxysmal dystonia; (NREM): non-REM;

(PAs): paroxysmal arousals; (PNES): psychogenic non-epileptic seizures; (PTSD): posttraumatic stress disorder; (QOL): quality of life; (RBD): REM sleep behavior disorder; (S-EEG): stereo-EEG; (SHE): sleep-related hypermotor epilepsy; (stage 1-REM): stage 1-REM with tonic EMG; (V-PSG): videopolysomnography

\section{Acknowledgements}

Part of this paper was also presented at the International Forum on "Abnormal Behaviors during Sleep" [Five lecturers presented: Drs. Clete A. Kushida (Stanford University, USA), Federica Provini (University of Bologna, Italy), Birgit Frauscher (McGill University, Canada), Carlos H. Schenck

(University of Minnesota, USA), and the author] at the 43rd Annual Meeting of the Japanese Society of Sleep Research held in Sapporo, Japan on July 14, 2018. The content of this paper is partially based on the significant discussions that took place among the lecturers. I wish to thank the lecturers for their insightful and constructive comments and suggestions.

Authors' contributions

The author read and approved the final manuscript. 


\section{Funding}

None.

\section{Availability of data and materials}

Not applicable.

Ethics approval and consent to participate

Not applicable.

\section{Consent for publication}

Not applicable.

\section{Competing interests}

The author has no conflict of interest to declare.

Received: 12 May 2019 Accepted: 9 December 2019

Published online: 06 February 2020

\section{References}

American Academy of Sleep Medicine. International classification of sleep disorders, 2nd edition. Diagnostic and coding manual. Westchester: American Academy of Sleep Medicine; 2005.

American Academy of Sleep Medicine. International classification of sleep disorders. 3rd ed. Darien: American Academy of Sleep Medicine; 2014.

American Sleep Disorders Association (Thorpy MJ. Chairman). International classification of sleep disorders: diagnostic and coding manual. Rochester: American Sleep Disorders Association; 1990.

Breen DP, Högl B, Fasano A, Trenkwalder C, Lang AE. Sleep-related motor and behavioral disorders: recent advances and new entities. Mov Disord. 2018;33: 1042-55. https://doi.org/10.1002/mds.27375.

Chen DK, Sharma E, LaFrance WC Jr. Psychogenic non-epileptic seizures. Curr Neurol Neurosci Rep. 2017;17:71. https://doi.org/10.1007/s11910-017-0781-7.

Chiba S. "Sleep epileptology" — a new field of sleep medicine and epileptology. Sleep Biol Rhythms. 2019;17:1-2. https://doi.org/10.1007/s41105-018-00200-1.

Ferri $\mathrm{R}$, Fantini ML, Schenck CH. The role of REM sleep without atonia in the diagnosis of REM sleep behavior disorder: past errors and new challenges. Sleep Med. 2014;15:1007-8. https://doi.org/10.1016/j.sleep.2014.05.006.

Gates JR, Ramani V, Whalen S, Loewenson R. Ictal characteristics of pseudoseizures. Arch Neurol. 1985;42:1183-7.

Gibbs SA, Proserpio P, Terzaghi M, Pigorini A, Sarasso S, Lo Russo G, et al. Sleep-related epileptic behaviors and non-REM-related parasomnias: insights from stereo-EEG. Sleep Med Rev. 2016;25:4-20. https://doi.org/10 1016/j.smrv.2015.05.002.

Halász P, Kelemen A, Szúcs A. The role of NREM sleep micro-arousals in absence epilepsy and in nocturnal frontal lobe epilepsy. Epilepsy Res. 2013;107:9-19. https://doi.org/10.1016/j.eplepsyres.2013.06.021.

Hatta K, Kishi Y, Wada K, Takeuchi T, Ito S, Kurata A, et al. Preventive effects of Suvorexant on delirium: a randomized placebo-controlled trial. J Clin Psychiatry. 2017;78:e970-9. https://doi.org/10.4088/JCP.16m11194.

Herman ST, Walczak TS, Bazil CW. Distribution of partial seizures during the sleepwake cycle: differences by seizure onset site. Neurology. 2001;56:1453-9.

Hishikawa Y. Disturbance of neural systems in delirium. Int Psychogeriatr. 1991;3: 409-14.

Ingravallo F, Poli F, Gilmore EV, Pizza F, Vignatelli L, Schenck CH, et al. Sleeprelated violence and sexual behavior in sleep: a systematic review of medical-legal case reports. J Clin Sleep Med. 2014;10:927-35. https://doi.org/ $10.5664 / \mathrm{jcsm} .3976$.

Inouye SK, Westendorp RG, Saczynski JS. Delirium in elderly people. Lancet. 2014; 383:911-22. https://doi.org/10.1016/S0140-6736(13)60688-1.

Iranzo A, Graus F, Clover L, Morera J, Bruna J, Vilar C, et al. Rapid eye movement sleep behavior disorder and potassium channel antibody-associated limbic encephalitis. Ann Neurol. 2006:59:178-81.

Janz D. Epilepsy and the sleep-waking cycle. In: Vinken PJ, Bruyn GW, editors. The epilepsies: handbook of clinical neurology. Amsterdam: North-Holland Publishing Company; 1974. p. 457-90.

Kojima T, Matsushima E, Ando K. Eyes and the mind. Tokyo: Japan Scientific Societies Press; 2000.

Krishnan P, Sinha S, Taly AB, Ramachandraiah CT, Rao S, Satishchandra P. Altered polysomnographic profile in juvenile myoclonic epilepsy. Epilepsy Res. 2014; 108:459-67. https://doi.org/10.1016/j.eplepsyres.2013.12.007.
Lawton G, Baker GA, Brown RJ. Comparison of two types of dissociation in epileptic and nonepileptic seizures. Epilepsy Behav. 2008;13:333-6.

Lugaresi E, Cirignott F. Hypnogenic paroxysmal dystonia: epileptic seizures or a new syndrome? Sleep. 1981;4:129-38. https://doi.org/10.1093/sleep/4.2.129.

Lugaresi E, Cirignotta F, Montagna P. Nocturnal paroxysmal dystonia. J Neurol Neurosurg Psychiatry. 1986;49:375-80. https://doi.org/10.1093/sleep/4.2.129.

Manni R, Terzaghi M, Zambrelli E. REM sleep behaviour disorder in elderly subjects with epilepsy: frequency and clinical aspects of the comorbidity. Epilepsy Res. 2007;77:128-33.

Mekky JF, Elbhrawy SM, Boraey MF, Omar HM. Sleep architecture in patients with juvenile myoclonic epilepsy. Sleep Med. 2017;38:116-21. https://doi.org/10.1016/j.sleep.2017.02.013.

Milanak ME, Zuromski KL, Cero I, Wilkerson AK, Resnick HS, Kilpatrick DG. Traumatic event exposure, posttraumatic stress disorder, and sleep disturbances in a National Sample of U.S. adults. J Trauma Stress. 2019;32:1422. https://doi.org/10.1002/jts.22360.

Miller LA, Ricci M, van Schalkwijk FJ, Mohamed A, van der Werf YD. Determining the relationship between sleep architecture, seizure variables and memory in patients with focal epilepsy. Behav Neurosci. 2016;130:316-24. https://doi.org/10.1037/bne0000127.

Montagna P. Nocturnal paroxysmal dystonia and nocturnal wandering. Neurology. 1992;42:61-7.

Montagna P, Sforza E, Tinuper P, Cirignotta F, Lugaresi E. Paroxysmal arousals during sleep. Neurology. 1990;40:1063-6.

Parrino L, De Paolis F, Milioli G, Gioi G, Grassi A, Riccardi S, et al. Distinctive polysomnographic traits in nocturnal frontal lobe epilepsy. Epilepsia. 2012a: 53:1178-84. https://doi.org/10.1111/j.1528-1167. 2012. 03502.x.

Parrino L, Ferri R, Bruni O, Terzano MG. Cyclic alternating pattern (CAP): the marker of sleep instability. Sleep Med Rev. 2012b;16:27-45. https://doi.org/10.1016/j.smrv.2011.02.003.

Plazzi G, Tinuper P, Montagna P, Provini F, Lugaresi E. Epileptic nocturnal wanderings. Sleep. 1995:18:749-56.

Provini F, Plazzi G, Montagna P, Lugaresi E. The wide clinical spectrum of nocturnal frontal lobe epilepsy. Sleep Med Rev. 2000:4:375-86.

Provini F, Plazzi G, Tinuper P, Vandi S, Lugaresi E, Montagna P. Nocturnal frontal lobe epilepsy. A clinical and polygraphic overview of 100 consecutive cases. Brain. 1999;122:1017-31.

Provini F, Tachibana N. Acute REM sleep behavior disorder. In: Schenck CH, Högl B, Videnovic A, editors. Rapid-eye-movement sleep behavior disorder. Cham: Springer International Publishing; 2018. p. 153-72.

Roshan S, Puri V, Chaudhry N, Gupta A, Rabi SK. Sleep abnormalities in juvenile myoclonic epilepsy-a sleep questionnaire and polysomnography based study. Seizure. 2017;50:194-201. https://doi.org/10.1016/j.seizure.2017.06.021.

Schenck CH, Bundlie SR, Ettinger MG, Mahowald MW. Chronic behavioral disorders of human REM sleep: a new category of parasomnia. Sleep. 1986:9:293-308.

Shouse MN. Mechanisms of sleep and arousal: relationship to epilepsy. In: Bazil CW, Malow BA, Sammaritano MR, editors. Sleep and epilepsy: the clinical spectrum. Amsterdam: Elsevier Science BV; 2002. p. 93-107.

Staner L. Sleep and anxiety disorders. Dialogues Clin Neurosci. 2003;5:249-58.

Sudbrack-Oliveira P, Lima Najar L, Foldvary-Schaefer N, da Mota Gomes M. Sleep architecture in adults with epilepsy: a systematic review. Sleep Med. 2019;53: 22-7. https://doi.org/10.1016/.j.sleep.2018.09.004.

Sunwoo JS, Cha KS, Byun JI, Kim TJ, Jun JS, Lim JA, et al. Abnormal activation of motor cortical network during phasic REM sleep in idiopathic REM sleep behavior disorder. Sleep. 2019; https://doi.org/10.1093/sleep/zsy227.

Szaflarski JP, Ficker DM, Cahill WT, Privitera MD. Four-year incidence of psychogenic nonepileptic seizures in adults in Hamilton county, $\mathrm{OH}$. Neurology. 2000;55:1561-3.

Tachibana M, Tanaka K, Hishikawa Y, Kaneko Z. A sleep study of acute psychotic states due to alcohol and meprobamate addiction. In: Weitzman ED, editor. Advances in sleep research, vol. 2. New York: Spectrum Publications; 1975. p. 177-205

Tamura Y, Chiba S, Takasaki H, Tabata K, Ishimaru Y, Ishimoto T. Biperideninduced delirium model in rats: a behavioral and electroencephalographic study. Brain Res. 2006;1115:194-9.

Terzaghi M, Sartori I, Mai R, Tassi L, Francione S, Cardinale F, et al. Sleep-related minor motor events in nocturnal frontal lobe epilepsy. Epilepsia. 2007;48:335-41.

Terzaghi M, Sartori I, Mai R, Tassi L, Francione S, Cardinale F, et al. Coupling of minor motor events and epileptiform discharges with arousal fluctuations in NFLE. Epilepsia. 2008;49:670-6. 
Tinuper P, Bisulli F. From nocturnal frontal lobe epilepsy to sleep-related hypermotor epilepsy: a 35-year diagnostic challenge. Seizure. 2017:44:87-92. https://doi.org/10.1016/j.seizure.2016.11.023.

Tinuper P, Bisulli F, Cross JH, Hesdorffer D, Kahane P, Nobili L, et al. Definition and diagnostic criteria of sleep-related hypermotor epilepsy. Neurology. 2016:86:1834-42.

Tinuper P, Bisulli F, Provini F, Lugaresi E. Familial frontal lobe epilepsy and its relationship with other nocturnal paroxysmal events. Epilepsia. 2010;51:51-3. https://doi.org/10.1111/j.1528-1167.2009.02446.x.

Tinuper P, Cerullo A, Cirignotta F, Cortelli P, Lugaresi E, Montagna P. Nocturnal paroxysmal dystonia with short-lasting attacks: three cases with evidence for an epileptic frontal lobe origin of seizures. Epilepsia. 1990;31:549-56.

Tinuper P, Lugaresi E. The concept of paroxysmal nocturnal dystonia. In: Bazil CW, Malow BA, Sammaritano MR, editors. Sleep and epilepsy: the clinical spectrum. Amsterdam: Elsevier Science BV: 2002. p. 277-82.

Tinuper P, Lugaresi E, Vigevano F, Berkovich SF. Nocturnal frontal lobe epilepsy. In: Gerrini R, Aicardi J, Andermann F, Hallett M, editors. Epilepsy and movement disorders in children. Cambridge: Cambridge University Press; 2002. p. 97-110.

Tinuper P, Provini F, Bisulli F, Lugaresi E. Hyperkinetic manifestations in nocturnal frontal lobe epilepsy. Semiological features and physiopathological hypothesis. Neurol Sci. 2005;26:210-4.

van Golde EG, Gutter T, de Weerd AW. Sleep disturbances in people with epilepsy; prevalence, impact and treatment. Sleep Med Rev. 2011;15:357-68. https://doi.org/10.1016/j.smrv.2011.01.002.

Wada JA. Nocturnal recurrence of brief, intensely affective vocal and facial expression with powerful bimanual, bipedal, axial, and pelvic activity with rapid recovery as manifestations of mesial frontal lobe seizure. Epilepsia. 1988;29:209.

Wada JA, Purves SJ. Oral and bimanual-bipedal activity as ictal manifestation of frontal lobe epilepsy. Epilepsia. 1984;25:668.

Waterman K, Purves SJ, Kosaka B, Strauss E, Wada JA. An epileptic syndrome caused by mesial frontal lobe seizure foci. Neurology. 1987;37:577-82. https://doi.org/10.1212/WNL.37.4.577.

Williamson PD, Spencer DD, Spencer SS, Novelly RA, Mattson RH. Complex partial seizures of frontal lobe origin. Ann Neurol. 1985;18:497-504. https://doi.org/10.1002/ana.410180413.

\section{Publisher's Note}

Springer Nature remains neutral with regard to jurisdictional claims in published maps and institutional affiliations.

Ready to submit your research? Choose BMC and benefit from:

- fast, convenient online submission

- thorough peer review by experienced researchers in your field

- rapid publication on acceptance

- support for research data, including large and complex data types

- gold Open Access which fosters wider collaboration and increased citations

- maximum visibility for your research: over $100 \mathrm{M}$ website views per year

At $\mathrm{BMC}$, research is always in progress.

Learn more biomedcentral.com/submissions 